\title{
EEG decoding of semantic category reveals distributed representations for single concepts
}

\author{
Brian Murphy ${ }^{\mathrm{a}}$, Massimo Poesio ${ }^{\mathrm{a}, \mathrm{b}}$, Francesca Bovolo $^{\mathrm{b}}$, \\ Lorenzo Bruzzone $^{\mathrm{b}}$, Michele Dalponte ${ }^{\mathrm{b}}$, Heba Lakany ${ }^{\mathrm{c}}$ \\ ${ }^{a}$ Centre for Mind/Brain Sciences, University of Trento \\ ${ }^{b}$ Department of Information Engineering and Computer Science, University of Trento \\ ${ }^{c}$ Bioengineering Unit, University of Strathclyde
}

\begin{abstract}
Here we present a collection of advanced data-mining techniques that allows the semantics of individual concepts to be decoded from single trials of EEG data. Neural activity was recorded while participants silently named images of mammals and tools, and category could be detected in single trials with an accuracy well above chance, both when considering data from single participants, and when group-training across participants. The pattern of classifications made by the algorithm confirmed that the neural patterns identified are due to conceptual category, and not any of a series of processing-related confounds. The time intervals, frequency bands and scalp locations that proved most informative for prediction permit physiological interpretation: the widespread activation shortly after appearance of the stimulus (from 100ms) is consistent both with accounts of multi-pass processing, and distributed representations of categories. These methods provide a new alternative to fMRI for fine-grained investigations of the conceptual lexicon.
\end{abstract}

\section{Introduction}

Recordings of brain activity from neuroimaging and electrophysiological techniques have established themselves as crucial sources of evidence in the study of semantic representations. Imaging studies (e.g. Martin and Chao, 2001; Chao et al., 2002) have 
provided ample evidence supporting hypotheses concerning the localization of conceptual knowledge derived from earlier work with patients displaying semantic category deficits (Warrington and Shallice, 1984; Caramazza and Mahon, 2003). Now computationally intensive methods are increasingly becoming the method of choice for analysing such data (Haxby et al., 2001; Haynes and Rees, 2006; Shinkareva et al., 2008). The low signal-to-noise ratio of neural recordings means that many regularities remain hidden and can only be detected through large-scale pattern analysis (Norman et al., 2006).

However, such methods have generally led to very high-level insights about conceptual representations, rather than to answers to the more fine-grained questions raised by studies in other paradigms, such as behavioural experiments (Quillian, 1967; Collins and Quillian, 1970; Rosch, 1973; Smith et al., 1974; Neely, 1991), judgements elicited from informants (McRae et al., 2005; Vinson and Vigliocco, 2008), language acquisition work (Mandler, 1992; Bloom, 2002), computational simulations (Plaut, 1995; McClelland and Rogers, 2003) or models derived from corpora (Lund et al., 1995; Landauer and Dumais, 1997; Rapp, 2004; Poesio and Almuhareb, 2005; Padó and Lapata, 2007; Baroni et al., 2010) - not to mention work on formal models such as Pustejovsky (1995); Kamp and Partee (1995). Most studies examine only very coarse-grained distinctions, for example comparing abstract and concrete concepts, verbs and nouns, or natural and artefactual kinds (Pulvermüller, 2002). A further limitation of this work is that the well-established differences seen between groups of stimuli representing natural and artefactual concepts (e.g. Kiefer, 2001; Chao et al., 2002) are compatible with a number of interpretations. Since the natural concepts investigated are predominantly animals and plants, and artefactual stimuli are typically small functional objects conventionally termed 'tools', several semantic distinctions provide plausible explanations: biological/non-biological entities, larger/smaller objects, moving/non-moving entities, sentient/non-sentient entities and manipulable/non-manipulable objects. More 
pessimistically, such effects could be due to confounding variables (e.g. lexical frequency, image complexity) that may vary systematically across stimulus groups. Finally, the statistics applied to neural data tend to identify single or small numbers of loci in the neural activity (at a precise location and approximate timing with fMRI; at a precise time and approximate location with EEG) that are activated differentially by each group of stimuli. This is despite the fact that recent experimental work demonstrates more widely spread and overlapping activations (e.g. Haxby et al., 2001; Pulvermüller, 2005; Mitchell et al., 2008), consistent with theories that favour distributed representations (Spitzer, 1999; Tyler and Moss, 2001; Barsalou, 2003).

We believe that large-scale, systematic explorations of the mental lexicon with neural data are necessary, involving both a more careful analysis of conceptual distinctions and a greater range of categories. However, the high cost of fMRI studies may make such systematic explorations impractical. Fortunately, the theory that conceptual knowledge is distributed would predict some form of synchronization between the relevant areas, of the kind that may be detected using EEG.

In this paper we detect which of two familiar semantic classes are being processed (land mammals or work tools, which should have similar conceptual representations across languages), by applying data mining and machine learning techniques to single trials of recorded EEG signals. The behavioural task was a simple one that requires activation of conceptual representations: silent naming of image stimuli, WHICH INVOLVES OBJECT RECOGNITION, lexical retrieval and sub-vocal language production. AS IS DISCUSSED IN THE FOLLOWING SECTION, TASKS THAT INVOLVE CONCEPTUAL PROCESSING HAVE BEEN SHOWN TO ELICIT NEURAL RESPONSES THAT PERSIST ACROSS TASKS IN THE VISUAL AND AUDITORY MODALITY, AND FOR IMAGE AND LEXICAL STIMULI, SUGGESTING THAT THEY ACCESS A NEXUS OF LINGUISTIC KNOWLEDGE AND PERCEPTUAL EXPERIENCE.

Our adaptive analyses search through the signal to identify the scalp locations, time 
intervals and frequency bands at which the categorial distinction is most clearly present. On this basis, we can predict the semantic category of a single stimulus presentation with an accuracy well above chance both when training and evaluation data come from the same participant (mean $72 \%, \mathrm{p}<0.001$, binomial test with chance at 0.5 ), and when they come from different participants (mean 61\%, p<0.001). By amalgamating predictions over multiple analyses, the categorial membership of single stimuli can be determined with an accuracy of $98 \%$. Crucially, since the analysis technique can classify single stimulus trials, a range of lexical, visual and task-related confounding variables can be excluded as possible explanations for the performance of the algorithm, allowing us to conclude that the activations that are identified correspond to conceptual distinctions rather than group differences in the cognitive load of perceptual processing, lexical search, or other task-related activity.

The paper proceeds as follows. The next section provides additional theoretical background, particularly on the relationship between the patterns identified by the algorithm and underlying brain physiology and function. Section 3 describes in detail the experimental paradigm used, and the analytical methods applied. Classification results are given in section 4, for analyses on individual participant sessions and groupprediction across participants, together with a regression model analysis to establish that the accuracies achieved are not due to confounding variables, whether singly or in combination. Finally, in the discussion section we consider the physical and functional interpretation of the results, and what future directions could be taken with this analytical paradigm.

\section{Background}

Early work on the neural instantiation of conceptual knowledge suggested that different kinds of semantic information are isolated in anatomically distinct areas of the brain. Work with brain damaged patients (Warrington and Shallice, 1984) found selective impairment of conceptual knowledge on animals and tools, leading to the hypoth- 
esis that sensory (primarily visual) and functional (conventional uses and associated behaviours) properties were located in different brain areas. Brain imaging work that followed (Martin et al., 1996) was consistent with the related proposal (Caramazza and Shelton, 1998) that animate and inanimate kinds were localised in different parts of cortex. Such studies also provided evidence that the location of these activations is largely consistent across individuals and across tasks (Martin and Chao, 2001).

More recent work however seems to indicate that the neural patterns associated with the representation of conceptual knowledge are more complex than such 'hotspot' models would suggest. Haxby et al. (2001) demonstrated that neural activity in marginal and overlapping areas of cortex, beyond the foci of activity identified by conventional statistical tests, could be used to correctly decode the semantic category of image stimuli presented during an fMRI session. Mitchell et al. (2008) showed that the meaning of concrete nouns could be decomposed into properties that held a predictive relationship with widely distributed activities in the brain.

MUCH OF THE WORK JUST CITED USES IMAGE STIMULI, AND SO THESE EFFECTS COULD BE INTERPRETED AS BEING DUE TO VISUAL REGULARITIES THAT ARE CONFOUNDED WITH SEMANTIC CLASS. BUT DISTRIBUTED CONCEPTUAL REPRESENTATIONS HAVE ALSO BEEN OBSERVED DURING PASSIVE LINGUISTIC TASKS THAT DID NOT INVOLVE VISUAL PRESENTATION (PUlVERMÜLLER, 2005). AND THE DISSOCIATIONS IN NEURAL ACTIVITY THAT ARE TYPICALLY SEEN DURING OBJECT RECOGNITION HAVE ALSO BEEN OBSERVED DURING READING OF WORD STIMUli, Both With PET (PeRAni et AL., 1999) AND FMRI (ChaO ET AL., 1999). The FACt that CONGENITALly Blind PARTicipants (MAHON ET AL., 2009) ALSO SHOW THESE PATTERNS, DEMONSTRATES THAT THIS CANNOT BE A SECONDARY EFFECT VISUAL EXPERIENCE EVOKED BY WORD STIMULI. FURTHER EVIDENCE FOR THE MODALITY-INDEPEDENCE OF THESE REPRESENTATIONS AT A FINER SPATIAL SCALE IS PROVIDED B y CONNOLLY AND HAXBy (2010), WhO 
SHOW THAT PATTERNS OF NEURAL ACTIVITY IN EARLY VISUAL AREAS RELFECT PERCEPTUAL ATTRIBUTES, THE VENTRAL TEMPORAL CORTEX ENCODES CONCEPTUAL DISTINCTIONS.

Such empirical work is consistent with recent theories that hold that conceptual knowledge is distributed, perhaps taking the form of a 'conceptual map' (Spitzer, 1999), of 'situated simulations' (Barsalou, 2003) or of a 'word web' (Pulvermüller, 2002), according to which concepts are represented in 'neuron webs' with distinct cortical topographies, AND WHERE CONCEPTUAL REPRESENTATIONS FORM A NEXUS BETWEEN PERCEPTUAL EXPERIENCE AND LINGUISTIC KNOWLEDGE.

This suggests the need for some form of synchronization between the firing of these neurons, of the kind that might be detected with EEG. Indeed Tallon-Baudry and Bertand (1999) make just such a proposal, and find evidence of event-related synchronisation in the gamma band $(\approx 40 \mathrm{~Hz})$ during object recognition. Here we are interested in differential activation to semantic classes, and category specific differences in spectral power have been found at lower frequencies (in the band 1-20 Hz) with MEG (Gilbert et al., 2009) for animals versus tools, and with EEG for faces versus other objects (Rousselet et al., 2007). ERP evidence of category specificity has been found at both early $(\approx 150 \mathrm{~ms}$, Rossion et al., 2003; Itier and Taylor, 2004) and late ( $\approx 400 \mathrm{~ms}$, Kiefer, 2001) intervals after onset during image processing tasks. Some work interprets the first interval as being tied to perceptual differences and the second to semantic processing - a conclusion corroborated by Paz-Caballero et al. (2006), who found that the early ERPs were modulated by task, while the later ones were not. While other research suggests that the first burst of activity is driven by higher-level conceptual differences - Rousselet et al. (2007) compare faces to visually balanced (image configuration, spectral frequencies, and brightness histograms) houses, meaning that low-level visual differences (form and texture) cannot account for the effects; and Rousselet et al. (2004) see the same effects for animals versus inanimate objects, meaning that they are not specific 
to the interpretation of human faces (e.g. determining identity, reading expressions).

As already noted, the majority of neuroimaging studies of semantic category rely on group effects, revealed with grand averages across trials and participants. In the case of EEG, this is due to the impoverished nature of the signal that can be detected at the scalp. Here, we use a discriminative data-mining technique developed for BrainComputer-Interaction purposes (Dalponte et al., 2007), to perform category-based classification of single trials. The algorithm first identifies the time interval, frequency band and combination of scalp locations in the EEG signals that best distinguish the categories. It is a supervised method in that a portion of the neural data, labelled for semantic category, is used for learning (the "training" data), and the remaining data is then used to test the quality of learning (the "evaluation" data) by comparing the true category labels to those predicted by the algorithm. The signal decomposition used (CSP, Koles et al., 1990) yields measures of signal spectral power that respond selectively to the stimulus (analogous to event related synchronisation/desynchronisation, or ERS/ERD; see Pfurtscheller and Lopes da Silva, 1999) ${ }^{2}$ and that correspond to synchronous neural sub-assemblies, or networks of sub-assemblies, that are functionally related to the processing or representation of semantic classes. A generic machine learning algorithm then uses these measures of spectral power to classify single stimulus trials that were excluded from the training phase.

\section{Methods}

\subsection{Participants}

Seven staff and post-graduate students at the University of Trento took part in the study, all native speakers of Italian. Five of the participants were male and two female (age range 25-33, mean 29). One identified herself as left-handed, and two as

\footnotetext{
${ }^{2}$ In conventional measures of signal power (ERS/ERD, or ERSP in the terminology of Delorme and Makeig, 2003) power estimates are calculated relative to a baseline preceding each epoch. In this work, power estimates are absolute measures for each epoch, and comparisons are made across epochs. Here these measures will be termed "spectral power".
} 
ambidextrous or of mixed-handedness. All had normal or corrected-to-normal vision. Participants received compensation of $€ 7$ per hour. The studies were conducted under the approval of the ethics committee at the University of Trento, and participants gave informed consent.

\subsection{Experimental Paradigm}

Participants were asked to perform a silent naming task on grey-scale images of 30 land-mammals and 30 work tools. Each stimulus was presented six times, for a total 360 trials, their order being randomized on each session. ${ }^{3}$ Participants sat in a relaxed upright position $60 \mathrm{~cm}$ from a computer monitor in reduced lighting conditions. Images were presented on a medium grey background and fell within a $10^{\circ}$ viewing angle. The task duration was split into four blocks and participants were given the choice to pause between each. The cumulative task time did not exceed 45 minutes.

Each trial began with the presentation of a fixation cross for $0.5 \mathrm{~s}$, followed by the stimulus image, a further fixation cross for $0.5 \mathrm{~s}$ and a blank screen for $2 \mathrm{~s}$. Participants were instructed to silently name the object represented in their native tongue (Italian), using the first appropriate label that came to mind, and to press the keyboard spacebar with the left-hand to indicate they had found an appropriate word. If participants could not think of a suitable label, they were asked not to make a response. The image remained on the screen until the participant responded, or until a time-out of three seconds was reached. Participants were asked to keep still during the task, and to avoid eye-movements and facial muscle activity in particular, except during the $2 \mathrm{~s}$ blank period.

Mean reaction times varied from $1 \mathrm{~s}$ (participant $\mathrm{C}$ ) to $1.8 \mathrm{~s}$ (participant $\mathrm{G}$ ), and nullresponses ranged from $1 \%$ to $7 \%$. After the experimental session, each participant was asked to complete a questionnaire, in which they recorded the names they used during

\footnotetext{
${ }^{3}$ The first three participants viewed a slightly larger set of images (42 animals, 44 tools) some of which were discarded in later experiments due to difficulty of naming. All analyses reported in this paper refer to the reduced set of 30 animals and 30 tools.
} 
the EEG session. In particular they were asked to indicate whether they were unable to identify any of the stimuli, could not find an appropriate name, or used multiple labels on the various presentations of a single stimulus. Naming agreement in this task ranged from $87 \%$ to $90 \%$.

\subsection{Materials}

As mentioned in the introduction, the semantic categories used in studies of this kind are often somewhat arbitrary and so can present problems for the interpretation of results. Here a set of 30 land mammals were chosen to be both non-domesticated and non-threatening, to avoid emotional confounds whether positive (e.g. pets) or negative (e.g. predators). Thirty hardware and garden implements were chosen as genuine work tools. Appropriate photographs were sourced from the internet, and normalised visually (see Figure S.5 in the supplementary materials): each image file measured 300 pixels square; the image proper was converted to grey-scale, superimposed on a homogeneous light-grey background and had maximal horizontal and vertical dimensions of 250 pixels; image contrast was normalised. ${ }^{4}$ The concepts represented are listed below.

Land Mammals ant-eater, armadillo, badger, beaver, bison, boar, camel, chamois, chimpanzee, deer, elephant, fox, giraffe, gorilla, hare, hedgehog, hippopotamus, ibex, kangaroo, koala, llama, mole, monkey, mouse, otter, panda, rhinoceros, skunk, squirrel, zebra (Italian formichiere, armadillo, tasso, castoro, bisonte, cinghiale, cammello, camoscio, scimpanzè, cervo, elefante, volpe, giraffa, gorilla, coniglio, riccio, ippopotamo, stambecco, canguro, koala, lama, talpa, scimmia, topo, lontra, panda, rinoceronte, puzzola, scoiattolo, zebra)

Work Tools Allen key, axe, chainsaw, craft-knife, crowbar, file, garden fork, garden trowel, hacksaw, hammer, mallet, nail, paint brush, paint roller, penknife, pick-axe, plaster trowel, pliers, plunger, pneumatic drill, power-drill, rake, saw,

\footnotetext{
${ }^{4}$ Using the ImageMagick function normalise; see http://www.imagemagick.org/.
} 
scissors, scraper, screw, screwdriver, sickle, spanner, tape-measure (Italian brugola, ascia, motosega, taglierino, piede di porco, lima, forcone, paletta, seghetto, martello, mazza, chiodo, pennello, rullo, coltellino svizzero, piccone, cazzuola, pinza, stura lavandini, martello pneumatico, trapano, rastrello, sega, forbici, spatola, vite, cacciavite, falce, chiave inglese, metro)

\subsection{Recording and Preprocessing}

The experiment was conducted at the CIMeC/DiSCoF laboratories at Trento University, using a 64-electrode Brain Vision BrainAmp system, recording at $500 \mathrm{~Hz}$. A wide-coverage montage based on the 10-20 system was used, with a single right earlobe reference, and ground at location AFz. Electrode impedances were generally kept below $10 k \Omega$. However, sessions including electrodes that exceeded this limit were still included in subsequent analysis, as the techniques used proved robust to such noise.

Data preprocessing was conducted using the EEGLAB package (Delorme and Makeig, 2003). The data was band-pass filtered at $1-120 \mathrm{~Hz}$ to remove slow drifts in the signal and high-frequency noise, and then down-sampled to $300 \mathrm{~Hz}^{5}$ An ICA analysis was next applied using the EEGLAB implementation of the Infomax algorithm (Makeig et al., 1996). Artefactual ICA components were then identified and removed by hand in each data-set. In all cases, eye-artefact components were removed - usually one component for vertical movements including blinks, and another for horizontal movements. In a few cases components were found that isolated $50 \mathrm{~Hz}$ electrical noise, or electrode specific noise (usually due to problems with impedance), but in general a conservative approach was taken, leaving such components in the signal to avoid the inadvertent removal of neural activity. It was not possible to remove muscle noise, as such activity was rarely isolated in a single component. However the adaptive analysis techniques used proved robust to both intermittent and continuous muscle noise.

\footnotetext{
${ }^{5}$ In some cases data was further filtered and re-sampled to lower rates (between $150 \mathrm{~Hz}$ and $100 \mathrm{~Hz}$ ) to reduce the computational memory requirements of the analysis, after exploratory analyses had determined that the higher frequencies thus excluded were not informative for classification.
} 


\subsection{Analysis}

The analysis method (Dalponte et al., 2007) consists of a time/frequency window search to identify an information-rich band and interval for the distinction of interest; a supervised decomposition (Common Spatial Patterns, or CSP - see Parra et al., 2005; Model and Zibulevsky, 2006; Philiastides et al., 2006 for examples of other applications to cognitive neuroscience) to extract components of whole-scalp synchronous activity that are sensitive to this class distinction; and a general purpose machine learning algorithm (Support-Vector Machine or SVM) that uses the resulting measures of spectral power to predict the semantic class of each trial. Individual trial epochs are arbitrarily allocated to one of $k$ interlaced partitions of equal size in a $k$-fold training/evaluation procedure (e.g. in a 3-fold partition, one third of the data is held out in turn for evaluation, and training is performed on the remaining two thirds).

The time/frequency window search was developed for Brain-Computer Interface (BCI) applications, achieving state-of-the-art performance on a competition task involving imagined hand and foot movements (Dalponte et al., 2007). The goal of the technique is to define the best combination of time and frequency intervals for the separation of the analysed categories. This method can be divided into two parts: a search strategy, and a computation of resulting class separability. The search strategy used in this paper was a grid-based region search: the time range $(0-500 \mathrm{~ms}$, relative to the appearance of the stimulus) and frequency range $(0-50 \mathrm{~Hz})$ of interest were divided into 15 intervals of equal length $(33 \mathrm{~ms}$ and $3.3 \mathrm{~Hz}$ respectively), to yield a total of 120 time spans $(0-33 \mathrm{~ms}, 0-67 \mathrm{~ms}, 0-100 \mathrm{~ms}, \ldots, 467-500 \mathrm{~ms})$ and 120 frequency spans $(0-3.3 \mathrm{~Hz}, 0-6.7 \mathrm{~Hz}, 0-10 \mathrm{~Hz}, \ldots, 46.7-50 \mathrm{~Hz})$, and all binary combinations of these spans were explored. For each of these 14,400 time/frequency windows, the signal data was band-pass filtered, cropped in time, and the class-labelled trials were processed with CSP to extract class-sensitive measures of spectral power (as described below). The computation of class-separability used the Bhattacharyya distance, a probabilistic mea- 
sure of cluster separation (Bhattacharyya, 1943; Bruzzone et al., 1995). Of all the windows examined, the combination of time and frequency spans that yields the largest separation between classes is considered the most informative window for classification purposes. To AVOID the DANGers of "Double-DipPing" (Pereira et Al., 2009; KRIEGESKORTE ET AL., 2009), THE WINDOWING PARAMETERS SELECTED FOR TESTING PREDICTION ACCURACY IN EACH PARTICIPANT WERE OPTIMIZED ON THE BASIS OF THE DATA FROM THE REMAINING SIX PARTICIPANTS ONLY.

The decomposition method used, CSP (Koles et al., 1990), can be considered similar to PCA or ICA, in that it extracts components of EEG activity that correspond to synchronous neural sub-assemblies. However it differs in that it is a supervised technique, in which the class-membership of each trial is also input to the algorithm. Thus, rather than extracting $C$ new component signals of whole-scalp activity (where $C$ is the number of input EEG channels) without regard to the task as PCA/ICA do, CSP extracts a series of components that are ranked by their sensitivity to the class-separation of interest, with an optimal variance for the two populations of EEG signals (i.e., high variance between classes and low variance within classes). In the considered case CSP extracts a set of $C$ components jointly ranked by the amplitude of the signal found in trials of the first class (Mammals, $\omega_{\text {Mammals }}$ ) and inversely by their signal amplitude during trials of the second class (Tools, $\omega_{\text {Tools }}$ ).

The CSP decomposition is calculated as follows. Let $E$ be a single trial of EEG signal, represented as a $C \times T$ matrix, where $C$ is the number of recording electrodes (64 in this case) and $T$ is the number of samples per epoch. The transformation matrix that permits the extraction of signal components specific to the classification is derived with the following steps:

1. Let $R$ be the normalized spatial covariance of a signal trial, defined as:

$$
R=\frac{E E^{T}}{\operatorname{trace}\left(E E^{T}\right)}
$$


2. Compute the average normalized spatial covariance matrices $\bar{R}_{\text {Tools }}$ and $\bar{R}_{\text {Mammals }}$ for trials $\omega_{\text {Tools }}$ and $\omega_{\text {Mammals }}$, respectively, by averaging over all the trials of the same class.

3. Compute the composite spatial covariance matrix as:

$$
\bar{R}_{C}=\bar{R}_{\text {Tools }}+\bar{R}_{\text {Mammals }}=U_{C} \lambda_{C} U_{C}^{T}
$$

where $U_{C}$ is the matrix of the eigenvectors and $\lambda_{C}$ is the diagonal matrix of the eigenvalues of $\bar{R}_{C}$.

4. Let $P$ be the whitening transformation matrix computed as:

$$
P=\sqrt{\lambda_{C}^{-1}} U_{C}^{T}
$$

5. Transform the matrices $\bar{R}_{\text {Tools }}$ and $\bar{R}_{\text {Mammals }}$ individually as:

$$
S_{\text {Tools }}=P \bar{R}_{\text {Tools }} P^{T}, \quad S_{\text {Mammals }}=P \bar{R}_{\text {Mammals }} P^{T}
$$

These two matrices share common eigenvectors, i.e. $S_{T o o l s}=U \lambda_{T o o l s} U^{T}$ and $S_{\text {Mammals }}=U \lambda_{\text {Mammals }} U^{T}$. Moreover the sum of the corresponding eigenvalues is always 1 , i.e. $\lambda_{\text {Tools }}+\lambda_{\text {Mammals }}=I$. Thus, the eigenvector with the largest eigenvalue for class $\omega_{T o o l s}$ has the smallest eigenvalue for class $\omega_{\text {Mammals }}$, and vice-versa.

6. Compute the spatial filter $(S F)$ to be used in the transformation of the signal, as:

$$
S F=U^{T} \cdot P
$$

7. Transform each trial EEG $E$ into the desired specific components:

$$
J=S F \cdot E
$$

The matrix $J$ represents the final output of the CSP algorithm. As is usually done in the literature (Ramoser et al., 2000), we select the first and the last rows of this matrix as the components that are most representative for the classes $\omega_{\text {Tools }}$ and $\omega_{\text {Mammals }}$, 
respectively. ${ }^{6}$ This procedure can be interpreted as extracting the event-related spectral activity (i.e. the relative event-related synchronisation) of two synchronous neural structures which have been found to have an optimally differential response to the stimulus categories of interest.

The final categorization step is based on a Support-Vector Machine (SVM) classifier (Boser et al., 1992; Vapnik, 1998), a state-of-the-art machine learning technique that has been widely adopted in recent years. Relative to other classifiers, it provides high classification accuracy and very good generalization; involves few control parameters; is not computationally intensive; and has proven effective in classification tasks where the number of training samples is limited (see Noble, 2006 for a non-technical introduction). The SVM input for each trial consisted of two numbers, derived as such from the pair of signal components produced by the CSP algorithm:

$$
x_{p}=\log \left(\operatorname{var}\left(J_{p}\right)\right)
$$

where $J_{p}$ is the $1 \times T$ vector representing the $p$-th signal component of $J$. Taking the variance of the vector produces a measure that is proportional to signal power, and the $\log$ function transforms the data to approximate a normal distribution (Ramoser et al., 2000). The resulting pair of measures represent modulations in spectral power which are sensitive to mammal and tool stimuli respectively:

$$
x=\left[\log \left(\operatorname{var}\left(J_{1}\right)\right), \log \left(\operatorname{var}\left(J_{M}\right)\right)\right]
$$

where $J_{1}$ and $J_{M}$ represent the first and the last components of $\mathrm{J}$, respectively. Features in $x$ were scaled to a range of -1 to +1 across all trials. The SVM implementation used was LIBSVM (Chang and Lin, 2001).

\footnotetext{
${ }^{6}$ Preliminary analyses using more than one component per class did not provide substantive improvement in classification accuracy. For example, over the seven individual analyses reported in section 4.2, taking two components per class gave an average 1 percentage point improvement in classification accuracy, while taking five components per class gave no improvement.
} 


\section{Results}

\subsection{Time/frequency interval optimisation}

As detailed in the previous section, a grid based region search was performed on the time-range $0-500 \mathrm{~ms}$ and the frequency range $0-50 \mathrm{~Hz}$, in steps of $33 \mathrm{~ms}$ and $3.3 \mathrm{~Hz}$ respectively. In total just under 15 thousand time/frequency windows were considered for each participant. For each window, the EEG signals were band-pass filtered, divided into epochs of the relevant time interval, and decomposed with CSP to identify class specific components of neural activity. The Bhattacharyya metric of class separability was computed on the spectral power measures between the set of Mammal trials and the set of Tool trials. This process was carried out separately for each of the seven subject sessions. As mentioned in the previous section, the time/frequency cropping parameters for each participant that yield the best separation between mammal and tool epochs constitute an optimum, maximising the size of the effect between classes.

Figure 1 (right panel) shows the time/frequency space for the EEG data from a single session (participant B). The value at each point on the graph is the mean Bhattacharyya distance for all windows that included that portion of the time/frequency space. As is apparent from the figure, the most informative interval runs from shortly after stimulus onset to around $350 \mathrm{~ms}$, and the theta $(4-8 \mathrm{~Hz})$ and lower alpha $(8-10 \mathrm{~Hz})$ bands are the most relevant frequency range.

\section{[Insert Figure 1]}

The optimal window selected according to the maximum separability in this case was $(100-370 \mathrm{~ms} ; 3-17 \mathrm{~Hz})$, indicated in black. For comparison, the next three nearoptimal solutions are indicated in white.

The left panel of Figure 1 shows the corresponding plot taking a mean over the spaces of all seven participants. Here the time and frequency intervals are broadly similar, starting slightly later, and extending somewhat into the low beta band. The optimal windows for each individual participant are shown in white and the mean optimal win- 
dows is shown in black: $95-360 \mathrm{~ms}$ and $4.1-18.3 \mathrm{~Hz}$. Individual time/frequency spaces for each participant are included in the supplementary materials (Figure S.3).

\subsection{Individual Classification}

FOR EACH PARTICIPANT, THE 64-CHANNEL EEG DATA WAS BAND-FILTERED AND EPOCHED ACCORDING TO AN AVERAGE OPTIMUM WINDOW COMPUTED OVER THE REMAINING SIX PARTICIPANTS. Using a 5-fold partition procedure, the 360 trials were split into five non-overlapping evaluation sets of equal size (72 trials, evenly split between each class). For each evaluation set, the remaining $4 / 5$ of the data served as the training set (288 trials). Accuracy was then computed by comparing the SVM predictions of membership to the true category of the image presented in each evaluation trial, over all five folds, on the basis of the spectral power measures extracted (the distribution of features extracted for a typical participant are included in the supplementary materials as Figure S.1). Default SVM settings were used: a radius basis function kernel with settings of $1 / n$ for the gamma value (where $n$ is the number of training data points, 288) and 1 for the cost parameter $C .{ }^{7}$ As Figure 2 shows, classification accuracy over single trials was significant for all participants $(\mathrm{p}<0.01)$ ranging from $57 \%$ to $80 \%$ (performance significantly above chance at $\alpha=0.05$ is indicated by a dotted line), with a mean of $72 \%(\mathrm{p}<0.001)$. Significance was calculated using a binomial test ( $n=360, p=0.5)$, and validated with a bootstrapping simulation (based on 1000 random permutations of category labels). ${ }^{8}$

\section{[Insert Figure 2]}

The CSP components extracted can be visualised by means of scalp-maps to give an indication of the distribution of the neural sub-assemblies that are informative for

\footnotetext{
${ }^{7}$ Usually, optimisation of SVM parameters can lead to considerable increases in classification performance. In our case, several tuning strategies were investigated, but did not improve results. We believe that this is because CSP is a powerful supervised learning technique that largely succeeds in linearly separating the training data, and so a ceiling is reached in cross-validation performance at the SVM.

${ }^{8}$ The distribution assumed by the binomial test had a mean and standard deviation over trials of 180 and 9.487 respectively. The permutation-based simulation yielded a mean and standard deviation of 179.9 and 9.58 trials.
} 
category. The precise distribution varied across participants, but a wide range of occipital, parietal and frontal areas played a role. The scalp maps from participant $\mathrm{C}$, shown in Figure 3 (top panel), are typical, showing activation distributed over all these areas. The mean activation maps over all participants are shown in the lower panel. Individual maps for all participants are available in the supplementary materials (Figure S.3).

\section{[Insert Figure 3]}

\subsection{Group Classification}

As discussed above, there are some differences between participants in the optimal time/frequency windows found, in the mammal and tool-specific spatial components extracted, and in the final categorisation accuracy achieved. This raises the question of to what extent category-specific neural activities are shared across participants. Crossparticipant generalisation in EEG work is complicated by many factors: the signal to noise ratio at each electrode is affected by the impedance achieved on the contact to the scalp, which is influenced by local differences in skin condition; the spatial location of electrodes relative to underlying cortex will vary according to the size and shape of the head; and as is also the case with other imaging technologies, there may be individual differences in brain anatomy or functional localisation across participants. While BrainComputer-Interaction tasks have demonstrated that generalisation is possible for the activity produced during motor planning and execution (see e.g. Curran and Stokes, 2003), it has not been established that activities associated with higher cognitive states generalise similarly well across participants.

The group analysis was carried out in a similar way to the individual analyses, using the same strategy for selecting an optimal time/frequency window. Since the signal to noise ratio varies across channels and across participants, all signals were normalised to $z$-score values (i.e. each channel from each participant was individually transformed so that it had a mean value of 0 and a standard deviation of 1). As before, the data was band-filtered and epoched, before being partitioned into evaluation and training 
sets. Here, a 7-fold partition procedure was used, where in each case the data from six participants (2160 trials) was used for training and the data from the left-out participant (360 trials) was used for evaluation. Accuracy was then computed by comparing the SVM predictions of category membership to the category of the image presented in each evaluation trial.

The results are illustrated in Figure 4. Classification accuracy was significant for all participant sessions $(\mathrm{p}<0.01)$, ranging from $56 \%$ to $68 \%$. The average accuracy was $61 \%$. The components isolated for group classification (Figure 5) overlap considerably with those for individual classification (Figure 3), but the information gained from frontal areas is reduced, and that from occipital areas is increased.

\section{[Insert Figures 4 and 5 here]}

\subsection{Discounting Nuisance Variables}

An obvious question about these results is whether they represent a successful detection of neural activity specific to semantic category, or they are rather driven by some lower level confound such as word length, ease of naming, or image complexity. Activity in the frequency bands identified here have been found to be modulated by a wide variety of cognitive tasks in verbal, visual and spatial processing (see reviews by Pfurtscheller and Lopes da Silva, 1999; and Kahana, 2006). Our stimuli were chosen to be reasonably frequent, visually identifiable and easily nameable, while constituting plausible categories that people use in everyday life and which are commonly lexicalised in human languages. No attempt was made to balance the materials for the many conceptual, visual and lexical confounds that have been raised in the literature, as we did not consider it feasible to find a set of stimuli that were suitable on the conceptual, visual and lexical levels, while also having comparable distributions on the values of all potential confounds. Instead, the trial-by-trial predictions made by the algorithm allow the contribution of any confound to be evaluated systematically at the analysis stage. 
An initial answer to the question of confounds is provided by the distribution over the scalp of the informative signal components. On average the depictions of mammals occupied a larger proportion of the screen display area and are more detailed, and so one possible alternative explanation of the results would be that we are detecting the additional perceptual processing that they demand. Cursory examination of the individual-level scalp-maps (Figure S.3, supplementary materials) shows that the informative activity is predominantly outside of the occipital areas where visual processing is focused, making this unlikely. But for the group analyses it remains a plausible explanation.

A further test is to look for patterns of errors in the classification. If the same items are repeatedly misclassified by the algorithm, it may be some confounding characteristic of the stimulus that is responsible. For example, if the analysis had identified components of neural activity that are sensitive to lexical length, and was using this to guess category membership (on average the mammal words were somewhat shorter than the tool words - see Table 1 below), then a mammal with a longer name (such as ippopotamo 'hippopotamus') should be consistently grouped with the tools, and a tool with a shorter name (e.g. sega 'saw') should be grouped with the mammals.

\section{[Insert Table 1 here]}

If on the other hand the classification errors are simply due to random noise, we would expect classification of stimuli to be more reliable after amalgamating predictions over multiple presentations. Here committee predictions are made by taking the majority classification over several trials (e.g. if a single stimulus was classified as a mammal on two presentations and as a tool on four presentations, the committee prediction is a tool). Making committee predictions separately for each participant does increase accuracy, represented by the grey bars on Figure 2. In all participants there is a noticeable improvement in accuracy, and the mean rate rises to $80 \%$ (grey bar on right). Similarly, taking the majority classification for each stimulus over all presen- 
tations to all participants raises classification accuracy to $98 \%$ (black bar on the far right), indicating that no single confound can account for the overall behaviour of the algorithm.

However, this does not exclude the possibility that some combination of confounding factors is being exploited by the algorithm. To investigate this, a regression model can be used to determine the relative contributions of a set of variables to the output of interest. In this case the output (the dependent variable, which we are trying to explain) is the committee category prediction for each stimulus, averaged over all presentations to all participants. The possible input variables (independent variables) are the true stimulus category, and a range of confounding variables related to the stimulus images, the words they represent, and task performance. ${ }^{9}$

The lexical variables considered were word length in syllables, and lexical log frequency calculated using a large web-collected corpus (Baroni et al., 2009). The possible visual confounds examined were image brightness, mean spatial frequency (a measure of low-level detail), and visual complexity (calculated using GIF and JPEG image compression - see Székely and Bates, 2000; Forsythe et al., 2008). Finally the three metrics of participant performance included were naming consistency, reaction time, and number of null reactions (i.e. how often a time-out was reached, because a participant could not think of an appropriate word within three seconds). Table 1 lists the variables, together with several measures of the degree of balance across the categories: the first column of numbers gives the probability that this variable is balanced across the two groups of stimuli, using a $t$-test; the second and third columns give respectively the rank correlation of each variable with the true category of the stimuli, and the linear correlation to the committee prediction produced by the algorithm. The

\footnotetext{
${ }^{9}$ Conceptual variables were not available for the Italian words used in the study, but concreteness is not expected to vary across the exclusively nominal object stimuli, and imagery should play no role in a picture naming task. Familiarity may play a role, and is indirectly reflected in the model as it co-varies strongly with lexical frequency (e.g. in the MRC Psycholinguistic Database, Wilson, 1988, there is a 0.74 rank correlation between familiarity and frequency).
} 
correlations were performed against the category of tools, so positive correlations indicate a larger value in the confound for tools, and a negative correlation indicates a larger value in the confound for mammals (e.g. the tool words were longer, and the mammal words were more frequent).

A linear regression model was then constructed using all these variables. ${ }^{10}$ All variables were $z$-score normed, so that the weights assigned to them by the regression model would be comparable. Care must be taken when selecting the inputs for such a model, as closely correlated variables can make the model unstable. For this reason image complexity was excluded, since it correlated very highly with image brightness $(r=0.92) .{ }^{11}$

The full model containing the true category value and all these possible confounds explained a very high amount of the variance $\left(r^{2}=0.81\right)$ in the predictions made in individual analyses. The relative contributions of each variable can be seen in Table 2. The first column shows that only two variables - the true stimulus category and image brightness - reach significance in explaining the behaviour of the algorithm in individual analyses. A reduced version of the model, where superfluous variables are automatically removed step-wise to improve overall fit, settles on these same two variables (figures in brackets). ${ }^{12}$ These weights suggest that category has over seven times the explanatory power of any of the confounds.

A linear model to explain the group analysis gave similar results (second column in Table 2). Despite a lower overall fit $\left(r^{2}=0.63\right)$, it again showed that category had many times more explanatory power than any of the possible confounds, which in this case were image detail (based on spatial frequencies) and word length (though this variable's contribution was not significant).

\footnotetext{
${ }^{10}$ Using the $\operatorname{lm}()$ function in $\mathrm{R}$.

${ }^{11}$ The linear models described below were replicated using image complexity in place of image brightness, and gave very similar results, with marginally lower measures of model fit.

${ }^{12}$ The step() function in $\mathrm{R}$ derives an optimal linear model by using the Akaike information criteria to incrementally remove variables.
} 


\section{[Insert Table 2 here]}

Finally, manual variations on these models can be built to compare the variance explained by subsets of variables. A model using all seven potential confounds but excluding the category of the stimuli explains only $51 \%$ and $34 \%$ of variance (for individual and group analyses respectively). Models using significant confounds only (image brightness for the individual model; and image detail for the group model) explained $51 \%$ and $15 \%$. A model using the true category alone accounts for $80 \%$ and $61 \%$ of the variance respectively - more of the algorithm's predictive behaviour than any of the confounds, individually or in combination.

All this evidence indicates that the category of the stimuli provides the best explanation for the behaviour of the algorithm. While the confounds can also model some of the algorithm's behaviour, this can be explained by their partial correlations with semantic class. The comparison of models with $\left(r^{2}=0.81\right.$ and 0.63$)$ and without $\left(r^{2}=0.80\right.$ and 0.61 ) confounds demonstrates that the information supplied by confounds is almost entirely superfluous.

\section{Discussion}

This is the first work to report the decoding of semantic categories from neural activity using EEG data, and also to take advantage of single-trial analyses to systematically exclude a range of low-level confounds as alternative explanations. Comparison with similar work using imaging data (Haxby et al., 2001, who achieves $85-100 \%$ accuracy on a larger number of categories) suggests that fMRI signals may be more informationrich in this respect, but we hope that continuing work on improving these techniques may close that gap. As EEG studies can be performed at much lower cost than fMRI, they may be a more feasible methodology for larger-scale investigations of the lexicon. We are now replicating these experiments with an expanded set of materials to establish the precise nature of the semantic distinction being detected, and with MEG to see to what extent it can improve individual and group-level prediction. 
The methods described in this paper show clear differences in the pattern of EEG signals observed during the processing of superordinate categories. However, the trained algorithm did not prove to be sensitive to conceptual relatedness within categories - for example that koala is more similar to kangaroo than either are to camel, and that garden fork and shovel are more closely related than they are to chainsaw. Ideally one could find correspondences between the semantic space described by this technique (see Figure S.2 in the supplementary materials for a graphical representation) and those derived from informants' judgements of typicality or semantic similarity (e.g. Morrow and Duffy, 2005; DeDeyne et al., 2008) or analogous measures of relatedness derived from language corpora and lexica (e.g. Lund et al., 1995; Lin, 1998; Patwardhan et al., 2003), which are of particular interest to us - but none of several comparisons of this kind that we carried out yielded significant results. This indicates that the discriminative classification techniques used are working as they are designed to, optimising the class distinction while ignoring all other aspects of the data. We are currently developing other data mining techniques that are better suited to extracting intra-categorial structure also (see Murphy et al., 2009 for a preliminary report on this work). Nevertheless, these trial-by-trial techniques make it possible for the first time to systematically exclude suspected confound variables (without exhaustive balancing of authentic materials, or the use of tailored fillers), and to potentially distinguish between the various semantic interpretations that can be attached to the group analyses reported in many neuroimaging and electrophysiological studies.

Currently we achieve highly significant classification for single participant data, and while generalising across individuals of a single language group, but we do not yet know if the neural patterns identified would generalise to other materials. Some preliminary analyses were also carried out based on earlier smaller scale experiments that used more heterogeneous materials (100 trials of mixed animals and mixed artefacts), to test if cross-language decoding (training on data from Italian speakers and evaluat- 
ing on data from English speakers) and cross-modal decoding (training on data from a silent image naming task and evaluating on data from a lexical visualisation task) is possible. While encouraging, these results only approached significance, and need to be replicated on a larger scale. In particular, we intend to use visual, rather than auditory presentation of lexical stimuli, to make cross-trial synchronisation more reliable (it is not straight-forward to determine at what point during each auditory word enough information has been received to allow categorisation to proceed). Lexical stimuli will also allow us to investigate more abstract categories than those that can be easily and unambiguously represented with images. Finally, it should be noted that the analysis presented here may represent either the process of categorisation, or the resultant representations, or both - though further analysis of the time-course of categorisation together with examination of later latencies may provide insights. Similarly with the current experimental design we cannot be conclusive about what phases of the image naming process (including object recognition and lexical retrieval) our algorithms take advantage of.

In terms of the neural realisation of semantics, we have identified scalp locations, time intervals and frequency bands that are especially informative about categorial differences. There is still no well-established consensus on the functional interpretation of variations in spectral power (see Pfurtscheller and Lopes da Silva, 1999; Kahana, 2006). The localised increases in spectral power seen here are conventionally interpreted as a reflection of a decrease in neural activity in some frequency bands ('cortical idling', in the alpha-band $\approx 8-12 \mathrm{~Hz}$ ), and a reflection of an increase in activity in others (theta, $4-8 \mathrm{~Hz}$; gamma $>20 \mathrm{~Hz}$ ), while the magnitude of the change in power is seen to be modulated by task difficulty or complexity (Jensen et al., 2007). More specifically, Pfurtscheller and Lopes da Silva (1999) describe upper alpha activity $(10-12 \mathrm{~Hz})$ as being linked to sensory and semantic processing, particularly in parietal-occipital areas, like those seen in this study. And Kahana (2006) report theta band activity (4-8Hz, also 
seen here) for verbal working memory tasks. The lowest frequencies $(<4 \mathrm{~Hz})$ may also reflect ERP components with a period of more than 250ms, such as N1/N170 effects.

Further, the latencies identified by our algorithms may seem rather early when compared to some of the ERP literature: the signals seen by Kiefer (2001) and PazCaballero et al. (2006) peak in separability after $300 \mathrm{~ms}$, and any earlier differences are interpreted as being due to perceptual processing. HOWEVER, THE TIME INTERVAL WE IDENTIFY ( $\approx 100-350 M S)$ IS MORE CONSISTENT WITH RECENT WORK THAT SUGGESTS THAT AT LEAST A FIRST PASS, COARSE CATEGORISATION IS CARRIED OUT MUCH EARLIER (SEE THORPE, 2009, FOR DISCUSSION): LIU ET AL. (2009) DETECTED CATEGORY SPECIFIC DIFFERENCES IN SINGLE TRIALS AS EARLY AS 100MS AFTER ONSET WITH INTERCRANIAL ELECTRODES, VISUAL INPUT CAN REACH THE FRONTAL LOBE AS EARLY AS 40-65MS AFTER ONSET (KIRCHNER ET AL., 2009), AND KIRCHNER AND THORPE (2006) FIND THAT PARTICIPANTS CAN MAKE AN EYE-MOVEMENT AS EARLY AS 120MS AFTER ONSET IN A CATEGORY DETECTION TASK. And shorter latencies for the semantic processing of words are well established: in a visual-world task Allopenna et al. (1998) show that eyemovements that depend on interpretation can begin as early as $200 \mathrm{~ms}$ after the auditory onset of a word, while the effect of semantics during lexical processing becomes apparent in EEG between 100 and 200ms (Pulvermüller, 2002, p.62).

The similarities to the time intervals (Rossion et al., 2003; Itier and Taylor, 2004; Rousselet et al., 2004) and spectral frequencies (Rousselet et al., 2007) seen in the faceprocessing literature are particularly striking. ${ }^{13}$ As discussed in the background section, it is unlikely that these effects are exclusively due to perceptual processing (they are seen when faces are compared to non-face stimuli that have been matched for low-level visual properties; and are also found for non-human animate stimuli). So while we cannot rule out that we are also detecting high-level visual categorisation processes, these

${ }^{13}$ We would like to thank the second reviewer for bringing these very relevant results to our attention. 
follow initial categorisation, and so are at a level of abstraction (e.g. having handles vs having legs; being animate/inanimate) where visual and linguistic descriptions are closely aligned. This conclusion stands, irrespective of one's assumptions as to whether the conceptual system has separate visual and linguistic modules or not. In this respect it is important that the concepts we have used as stimuli are highly imageable, as the linguistic or psychological features that are most salient (e.g. according to feature norms such as McRae et al., 2005, or as might be yielded by corpus-based models of semantics like Poesio and Almuhareb, 2008; Baroni et al., 2010), are predominantly ones that are easily visualisable (colour, shape, size of ears, number of legs, etc).

The scalp locations at which these differences are maximal point to brain regions in which the processing load is modulated by semantic category. It is difficult to draw precise anatomical conclusions on the basis of this data as there is no principled basis on which to decide on the number of dipoles to expect in a source localisation analysis, nor whether the point-sources such a model assumes are appropriate when interpreting distributed activations. But it is clear that semantic processing and the resulting representations are widely spread across the brain, in a fashion that is somewhat shared between the participants that took part in the study. In particular, the extensive dorsal activations seen can be interpreted an effect of motor planning induced by the visual processing of manipulable objects (Mishkin and Ungerleider, 1982; Goodale and Milner, 1992), or rather a reflection of conceptual representations proper, as have been suggested more recently both in the literature on visual processing (Almeida et al., 2008) and lexical comprehension (Pulvermüller, 2005). In the case of the second interpretation, this constitutes further support for theories of neuronal networks of word meanings that arise from our experience of the world and language (Spitzer, 1999; Tyler and Moss, 2001; Barsalou, 2003). 


\section{Acknowledgements}

We are very grateful to Lisandro Kaunitz, Francesco Vespignani, Laura Tonelli and Stefano Bertamini for assistance in data collection and analysis. We would also like to thank the three reviewers who gave very helpful observations and suggestions on the earlier draft of this paper. The work described here was funded by CIMeC, the Autonomous Province of Trento, and the Fondazione Cassa Risparmio Trento e Rovereto.

\section{References}

Allopenna, P. D., Magnuson, J. S., Tanenhaus, M. K., 1998. Tracking the time course of spoken word recognition using eye movements: Evidence for continuous mapping models. Journal of Memory and Language 38 (4), 419-439.

Almeida, J., Mahon, B. Z., Nakayama, K., Caramazza, A., 2008. Unconscious processing dissociates along categorical lines. Proceedings of the National Academy of Sciences of the United States of America 105 (39), 15214-15218.

Baroni, M., Bernardini, S., Ferraresi, A., Zanchetta, E., 2009. The WaCky wide web: a collection of very large linguistically processed web-crawled corpora. Language Resources and Evaluation 43 (3), 209-226.

Baroni, M., Murphy, B., Barbu, E., Poesio, M., 2010. Strudel: A corpus-based semantic model based on properties and types. Cognitive Science 34 (2), 222-254.

Barsalou, L. W., 2003. Situated simulation in the human conceptual system. Language and Cognitive Processes 18, 513-562.

Bhattacharyya, A., 1943. On a measure of divergence between two statistical populations defined by probability distributions. Bulletin of the Calcutta Mathematical Society $35,99-109$. 
Bloom, P., 2002. How Children Learn the Meaning of Words. MIT Press, Cambridge.

Boser, B. E., Guyon, I. M., Vapnik, V. N., 1992. A training algorithm for optimal margin classifiers. In: Haussler, D. (Ed.), 5th Annual ACM Workshop on COLT. ACM Press, Pittsburgh, pp. 144-152.

Bruzzone, L., Roli, F., Serpico, S. B., 1995. An extension of the Jeffreys-Matusita distance to multiclass cases for feature selection. IEEE Transactions on Geoscience and Remote Sensing 33 (6), 1318-1321.

Caramazza, A., Mahon, B. Z., 2003. The organization of conceptual knowledge: The evidence from category-specific semantic deficits. Trends in Cognitive Sciences 7 , $354-361$.

Caramazza, A., Shelton, J., 1998. Domain-specific knowledge systems in the brain: the animate-inanimate distinction. Journal of Cognitive Neuroscience 10, 1-34.

Chang, C.-C., Lin, C.-J., 2001. LIBSVM: a library for support vector machines.

Chao, L., Haxby, J., Martin, A., 1999. Attribute-based neural substrates in temporal cortex for perceiving and knowing about objects. Nature Neuroscience 2, 913-919.

Chao, L. L., Weisberg, J., Martin, A., 2002. Experience-dependent modulation of category related cortical activity. Cerebral Cortex 12, 545-551.

Collins, A. M., Quillian, M. R., 1970. Facilitating retrieval from semantic memory: The effect of repeating part of an inference. In: Sanders, A. F. (Ed.), Attention and Performance III. Vol. 33 of Acta Psychologica. North-Holland, Amsterdam, pp. 304314.

Connolly, A., Haxby, J., 2010. Similarity-based multi-voxel pattern analysis reveals an emergent taxonomy of animal species along the object vision pathway. Journal of Vision 10 (7), 964. 
Curran, E. A., Stokes, M. J., 2003. Learning to control brain activity: A review of the production and control of EEG components for driving brain-computer interface (BCI) systems. Brain and Cognition 51 (3), 326 - 336.

Dalponte, M., Bovolo, F., Bruzzone, L., 2007. Automatic selection of frequency and time intervals for classification of EEG signals. Electronics Letters 43, 1406-1408.

DeDeyne, S., Verheyen, S., Ameel, E., Vanpaemel, W., Dry, M. J., Voorspoels, W., Storms, G., 2008. Exemplar by feature applicability matrices and other Dutch normative data for semantic concepts. Behavior Research Methods 40 (4), 1030-1048.

Delorme, A., Makeig, S., 2003. EEGLAB: an open source toolbox for analysis of single-trial dynamics including independent component analysis. Journal of Neuroscience Methods 134, 9-21.

Forsythe, A., Mulhern, G., Sawey, M., 2008. Confounds in pictorial sets: the role of complexity and familiarity in basic-level picture processing. Behaviour Research Methods 40 (1), 116-129.

Gilbert, J., Shapiro, L., Barnes, G., 2009. Processing of living and nonliving objects diverges in the visual processing system: evidence from MEG. In: Proceedings of the Cognitive Neuroscience Society Annual Meeting.

Goodale, M. A., Milner, A. D., 1992. Separate visual pathways for perception and action. Trends in Neuroscience 15, 20-25.

Haxby, J., Gobbini, M., Furey, M., Ishai, A., Schouten, J., Pietrini, P., 2001. Distributed and overlapping representations of faces and objects in ventral temporal cortex. Science 293 (5539), 2425-30.

Haynes, J. D., Rees, G., July 2006. Decoding mental states from brain activity in humans. Nature Reviews Neuroscience 7 (7), 523-534. 
Itier, R., Taylor, M., 2004. N170 or N1? Spatiotemporal differences between object and face processing using ERPs. Cerebral Cortex 14 (2), 132.

Jensen, O., Kaiser, J., Lachaux, J.-P., 2007. Human gamma-frequency oscillations associated with attention and memory. Trends in Neurosciences 30 (7), 317-324.

Kahana, M. J., 2006. The cognitive correlates of human brain oscillations. The Journal of Neuroscience 26, 1669-1672.

Kamp, H., Partee, B., 1995. Prototype theory and compositionality. Cognition 57 (2), 129-191.

Kiefer, M., 2001. Perceptual and semantic sources of category-specific effects in object categorization: Event-related potentials during picture and word categorization. Memory and Cognition 29 (1), 100-116.

Kirchner, H., Barbeau, E., Thorpe, S., Regis, J., Liegeois-Chauvel, C., 2009. Ultrarapid sensory responses in the human frontal eye field region. Journal of Neuroscience 29 (23), 7599-7606.

Kirchner, H., Thorpe, S. J., 2006. Ultra-rapid object detection with saccadic eye movements: Visual processing speed revisited. Vision Research 46 (11), 1762-1776.

Koles, Z. J., Lazar, M. S., Zhou, S. Z., 1990. Spatial patterns underlying population differences in the background EEG. Brain Topography 2 (4), 275-284.

Kriegeskorte, N., Simmons, W., Bellgowan, P., Baker, C., 2009. Circular analysis in systems neuroscience: the dangers of double dipping. Nature Neuroscience 12 (5), $535-540$.

Landauer, T., Dumais, S., 1997. A solution to Plato's problem: the latent semantic analysis theory of acquisition, induction, and representation of knowledge. Psychological Review 104 (2), 211-240. 
Lin, D., 1998. Automatic retrieval and clustering of similar words. In: Proceedings of COLING-ACL. pp. 768-774.

Liu, H., Agam, Y., Madsen, J. R., Kreiman, G., April 2009. Timing, timing, timing: Fast decoding of object information from intracranial field potentials in human visual cortex. Neuron 62 (2), 281-290.

Lund, K., Burgess, C., Atchley, R., 1995. Semantic and associative priming in high dimensional semantic space. In: Proceedings of the 17th Cognitive Science Society Meeting. pp. 660-665.

Mahon, B., Anzellotti, S., Schwarzbach, J., Zampini, M., Caramazza, A., 2009. Category-specific organization in the human brain does not depend on visual experience. Neuron 63 (3), 397-405.

Makeig, S., Bell, A. J., Jung, T., Sejnowski, T. J., 1996. Independent component analysis of electroencephalographic data. In: Advances in Neural Information Processing Systems. Vol. 8. MIT Press, pp. 145-151.

Mandler, J. M., 1992. How to build a baby II: Conceptual primitives. Psychological Review 99, 587-604.

Martin, A., Chao, L., 2001. Semantic memory and the brain: structure and processes. Current Opinions in Neurobiology 11, 194-201.

Martin, A., Wiggs, C. L., Ungerleider, L. G., Haxby, J. V., February 1996. Neural correlates of category-specific knowledge. Nature 379, 649-652.

McClelland, J. L., Rogers, T. T., 2003. The parallel distributed processing approach to semantic cognition. Nature Reviews Neuroscience 4, 310-322.

McRae, K., Cree, G. S., Seidenberg, M. S., McNorgan, C., November 2005. Semantic feature production norms for a large set of living and nonliving things. Behavior Research Methods, Instruments, \& Computers 37 (4), 547-559. 
Mishkin, M., Ungerleider, L. G., Sep 1982. Contribution of striate inputs to the visuospatial functions of parieto-preoccipital cortex in monkeys. Behavioral Brain Research $6(1), 57-77$.

Mitchell, T. M., Shinkareva, S. V., Carlson, A., Chang, K.-M., Malave, V. L., Mason, R. A., Just, M. A., 2008. Predicting human brain activity associated with the meanings of nouns. Science 320, 1191-1195.

Model, D., Zibulevsky, M., 2006. Learning subject-specific spatial and temporal filters for single-trial EEG classification. NeuroImage 32 (4), 1631-1641.

Morrow, L. I., Duffy, M. F., 2005. The representation of ontological category concepts as affected by ageing: Normative data and theoretical implications. Behavior Research Methods 37 (4), 608-625.

Murphy, B., Baroni, M., Poesio, M., 2009. EEG responds to conceptual stimuli and corpus semantics. In: Proceedings of the Conference on Empirical Methods in Natural Language Processing. The Association for Computational Linguistics, pp. 619-627.

Neely, J. H., 1991. Semantic priming effects in visual word recognition: A selective review of current findings and theories. In: Besner, D., Humphreys, G. W. (Eds.), Basic Processes in Reading: Visual Word Recognition. Erlbaum, Mahwah, pp. 264336.

Noble, W. S., 2006. What is a support vector machine? Nature Biotechnology 24, $1565-1567$.

Norman, K. A., Polyn, S. M., Detre, G. J., Haxby, J. V., 2006. Beyond mind-reading: multi-voxel pattern analysis of fMRI data. Trends in Cognitive Sciences 10 (9), 424430.

Padó, S., Lapata, M., 2007. Dependency-based construction of semantic space models. Computational Linguistics 33 (2), 161-199. 
Parra, L. C., Spence, C. D., Gerson, A. D., Sajda, P., 2005. Recipes for the linear analysis of EEG. NeuroImage 28, 326-341.

Patwardhan, S., Banerjee, S., Pedersen, T., 2003. Using measures of semantic relatedness for word sense disambiguation. In: Proceedings of the Fourth International Conference on Intelligent Text Processing and Computational Linguistics. Mexico City, pp. 241-257.

Paz-Caballero, D., Cuetos, F., Dobarro, A., January 2006. Electrophysiological evidence for a natural/artifactual dissociation. Brain Research 1067 (1), 189-200.

Perani, D., Schnur, T., Tettamanti, M., Gorno-Tempini, M., Cappa, S. F., Fazio, F., 1999. Word and picture matching: a PET study of semantic category effects. Neuropsychologia 37 (3), 293-306.

Pereira, F., Mitchell, T., Botvinick, M., 2009. Machine learning classifiers and fMRI: a tutorial overview. Neuroimage 45 (1), S199-S209.

Pfurtscheller, G., Lopes da Silva, F. H., 1999. Event-related EEG/MEG synchronization and desynchronization: Basic principles. Clinical Neurophysiology 110, 18421857.

Philiastides, M., Ratcliff, R., Sajda, P., 2006. Neural representation of task difficulty and decision making during perceptual categorization: a timing diagram. Journal of Neuroscience 26 (35), 8965.

Plaut, D., 1995. Semantic and associative priming in a distributed attractor network. In: Proceedings of the Seventeenth Annual Conference of the Cognitive Science Society. pp. $37-42$.

Poesio, M., Almuhareb, A., 2005. Identifying concept attributes using a classifier. In: Proceedings of the ACL Workshop on Deep Lexical Semantics. pp. 18-27. 
Poesio, M., Almuhareb, A., 2008. Extracting concept descriptions from the Web: The importance of attributes and values. In: Buitelaar, P., Cimiano, P. (Eds.), Bridging the gap between text and knowledge. IOS, Amsterdam, pp. 29-44.

Pulvermüller, F., 2002. The neuroscience of language: on brain circuits of words and serial order. Cambridge University Press, Cambridge.

Pulvermüller, F., 2005. Brain mechanisms linking language and action. Nature Reviews Neuroscience 6, 576-582.

Pustejovsky, J., 1995. The Generative Lexicon. MIT Press, Cambridge.

Quillian, M. R., 1967. Word concepts: A theory and simulation of some basic semantic capabilities. Behavioral Science 12 (5), 410-430.

Ramoser, H., Gerking, J. M., Pfurtscheller, G., 2000. Optimal spatial filtering of single trial EEG during imagined hand movement. IEEE Transactions on Rehabilitation Engineering 8 (4), 441-446.

Rapp, R., 2004. A freely available automatically generated thesaurus of related words. In: Proceedings of LREC 2004. pp. 395-398.

Rosch, E., 1973. Natural categories. Cognitive Psychology 7, 328-350.

Rossion, B., Joyce, C., Cottrell, G., Tarr, M., 2003. Early lateralization and orientation tuning for face, word, and object processing in the visual cortex. Neuroimage 20 (3), $1609-1624$.

Rousselet, G., Husk, J., Bennett, P., Sekuler, A., 2007. Single-trial EEG dynamics of object and face visual processing. Neuroimage 36 (3), 843-862.

Rousselet, G., Mace, M., Fabre-Thorpe, M., 2004. Animal and human faces in natural scenes: How specific to human faces is the N170 ERP component? Journal of Vision $4(1), 13-21$. 
Shinkareva, S., Mason, R., Malave, V., Wang, W., Mitchell, T. M., Just, M. A., 2008. Using fMRI brain activation to identify cognitive states associated with perception of tools and dwellings. PloS ONE 3 (1).

Smith, E. E., Shoben, E. J., Rips, L. J., 1974. Structure and process in semantic memory: A featural model for semantic decisions. Psychological Review 81 (3), 214241.

Spitzer, M., 1999. The mind within the net: models of learning, thinking and acting. MIT Press, Cambridge.

Székely, A., Bates, E., 2000. Objective visual complexity as a variable in studies of picture naming. Tech. Rep. 12-2, Center for Research in Language Newsletter, University of California, San Diego.

Tallon-Baudry, C., Bertand, O., 1999. Oscillatory gamma activity in humans and its role in object representation. Trends in Cognitive Science 3 (4), 151-162.

Thorpe, S. J., 2009. The speed of categorization in the human visual system. Neuron $62(2), 168-170$.

Tyler, L. K., Moss, H. E., 2001. Towards a distributed account of conceptual knowledge. Trends in Cognitive Sciences 5 (6), 244-252.

Vapnik, V. N., 1998. Statistical Learning Theory. Wiley.

Vinson, D., Vigliocco, G., 2008. Semantic feature production norms for a large set of objects and events. Behavior Research Methods 40 (1), 183-190.

Warrington, E. K., Shallice, T., 1984. Category specific semantic impairments. Brain 107 (3), 829-853. 
Wilson, M. D., 1988. The MRC psycholinguistic database: Machine readable dictionary, version 2. Behavioural Research Methods, Instruments and Computers 20 (1), 6-11. 